\title{
Less isn't always more
}

\author{
OLKE C. UHLENBECK
}

Department of Biochemistry, Molecular Biology, Cell Biology and Chemistry, Northwestern University, Evanston, Illinois 60208, USA

A favorite experiment of RNA scientists is the "cutdown" or "boundary" experiment where an RNA of interest is truncated from its $5^{\prime}$ or $3^{\prime}$ end and the function of the smaller molecules evaluated. Such experiments often reveal that only a small part of a large RNA molecule is needed for the function of interest. In addition, by reducing the number of alternative conformational states that can plague RNA preparations, truncated RNAs that emerge from boundary experiments are often more active than the original molecule. A common complication in the interpretation of boundary experiments arises from the general way in which RNAs fold into their active structures. As we all know, RNA secondary structure elements often form between two or more parts of the molecule that are quite distant on the primary sequence. A boundary experiment performed on an RNA containing a critical long-range secondary structure element will identify as essential all of the RNA between the $3^{\prime}$-most and $5^{\prime}$-most parts of the element, even if much of the internal sequence is not important for function. This complication can be resolved by comparing several active sequences, deducing a consensus secondary structure, and preparing an appropriate internal deletion. This classic combination of cutdowns and internal deletions has been used to define the minimal RNA binding sequences of many different proteins and small molecules, establish RNA regulatory regions and define catalytic RNA motifs.

A more subtle complication encountered in such reductionist experiments is that complex folded RNAs often consist of a conserved core surrounded by one or more independently folding domains that stabilize the core through the formation of tertiary interactions. Because the role of these "buttressing" domains is primarily to provide stability, they are often not conserved and do not always score in boundary experiments. A good example of this situation is

Keywords: RNA catalysis; hammerhead; ribozyme; viroid; RNA structure

Reprint requests to: Olke C. Uhlenbeck, Department of Biochemistry, Molecular Biology, Cell Biology and Chemistry, Northwestern University, 2205 Tech Dr., Hogan 2-100, Evanston, IL 60208, USA; e-mail: o-uhlenbeck@northwestern.edu.

Article and publication are at http://www.rnajournal.org/cgi/doi/ 10.1261/rna.5155903. the P5abc subdomain in the Tetrahymena Group I intron. This subdomain, conserved only within a subgroup of these catalytic RNAs, folds back and stabilizes the conserved catalytic core. When P5abc is deleted, Group I splicing is slower because the active site is not as tightly packed (Engelhardt et al. 2000).

A striking recent example of the pitfalls of "RNA reductionism" surfaces in the paper of Khvorova et al. ( 2003) on the well studied hammerhead ribozyme. The hammerhead is a motif embedded in the RNA genomes of numerous plant pathogens and is responsible for the self-cleavage of the genome during replication (Symons 1992). Based on sequence comparisons, boundary experiments, and internal deletions done more than 15 years ago, the hammerhead was defined as three nonconserved RNA helices intersecting in a highly conserved catalytic core of 15 residues (Fig. 1). When the hammerhead was assayed in vitro, rapid and complete cleavage was observed at a unique site in the core that corresponded precisely to the site seen for the complete RNA genome in vivo. The hammerhead's small size and easily detected cleavage activity made it a popular molecule for study. Numerous biochemical and biophysical experiments on the hammerhead appeared, including several X-ray crystal structures (Pley et al. 1994; Scott et al. 1995). Prior to the discovery of RNAi, the hammerhead was also a popular tool to specifically cleave mRNAs inside cells, resulting in gene inactivation. Remarkably, it is now clear from the work of Khvorova et al. (2003) that virtually all hammerhead research since the mid-1980s (involving hundreds of papers) was performed on a motif that was smaller than what is needed for optimal activity. In other words, the hammerhead we have been working on had been truncated too far.

The initial clues that led Khvorova et al. (2003) to think that the hammerhead was actually larger than previously thought were experiments that embedded various hammerheads in the $3^{\prime}$ untranslated region of a reporter gene and then monitored gene activity in transiently transfected cells. When hammerhead sequences derived from fragments of several different viroids were inserted, gene inactivation was observed, presumably as a result of intramolecular cleavage and subsequent degradation of the reporter mRNA. However, when derivatives of the conventional hammerhead 

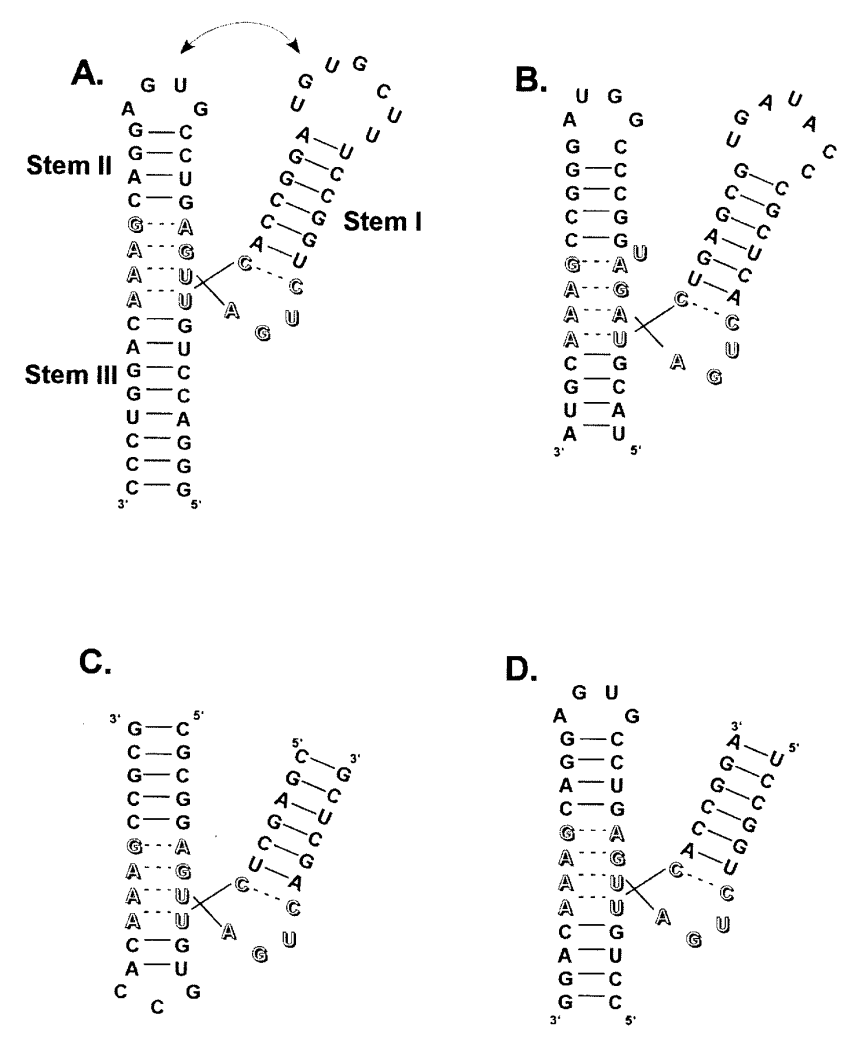

FIGURE 1. Secondary structures of hammerheads arranged to resemble the crystal structure. The catalytic core residues are indicated by open letters. $(A)$ The rapidly cleaving sequence derived from the satellite RNA of tobacco ringspot virus studied by Khvorova et al. (2003). The proposed tertiary interaction occurs between the loops at the ends of Stems 1 and 2. (B) The 55-nucleotide minimal hammerhead derived from the virusoid of leucerne transient streak virus identified by Forster and Symons. Hammerheads formed from two oligonucleotides described by $(C)$ Uhlenbeck (1987) and $(D)$ Haseloff and Gerlach (1988).

were inserted, no gene inactivation was observed. Thus, natural hammerheads worked in vivo, whereas nonnatural ones did not. An extensive mutagenic analysis of one natural hammerhead revealed that it contained essential sequence elements in the nonconserved loops at the ends of Stem 1 and Stem 2 (see Fig 1). A subsequent "swap" experiment with another natural hammerhead strongly suggested that the two loops interact to form critical tertiary interactions that had been missed by previous studies. Because the X-ray structures and several different solution experiments on conventional hammerheads showed that Stem 1 and Stem 2 were close in space, the introduction of a new tertiary interaction that connects them made sense. Indeed, the existence of an interaction between loop1 and loop2 through the formation of a pseudoknot had been proposed for several natural hammerheads (Miller and Silver 1991).

In vitro intramolecular cleavage experiments provided an explanation for the enhanced activity of the natural hammerheads. Although minimal hammerheads require relatively high nonphysiological magnesium ion concentrations to achieve a maximal rate of cleavage $(10-50 \mathrm{mM})$, the natural hammerheads were found to be remarkably efficient in the much lower magnesium concentrations typically found inside cells $(<1 \mathrm{mM})$. Indeed, the natural hammerheads cleave so rapidly in vitro under physiological conditions that it was not possible to accurately measure the rate using manual mixing methods $(>2 / \mathrm{min})$. Cleavage experiments in lower $\mathrm{pH}$ buffers suggest that in addition to cleaving at lower magnesium concentrations, natural hammerheads have a higher cleavage rate at saturating magnesium ion concentration (A. Khvorova, pers. comm.).

One of the puzzling aspects of hammerhead research has been that some of the biochemical data on chemically modified hammerheads seem inconsistent with the X-ray crystal structures. The inconsistencies took several forms. In some cases, hammerheads which were missing functional groups on a single nucleotide were unable to cleave despite the finding that the X-ray structure showed that the functional group protruded into the solvent, with no obvious role (McKay 1996). In other cases, the crystal structure showed a tertiary hydrogen bond between two functional groups, and although the cleavage rate decreased when one of the two interacting groups was disrupted, disrupting the other group had no effect on cleavage. Such disagreements were particularly disquieting in light of the excellent agreement between the crystal structures and functional group modification data for other small catalytic RNAs (Ryder and Strobel 2002). Wang et al. (1999) suggested that this structure-function dilemma could be explained if the structure of the catalytically active hammerhead was different than that in the crystal structures, perhaps as a result of a docking between two subdomains of the catalytic core. They were led to this proposal by biochemical experiments that concluded that a single divalent metal ion needed to stabilize the active conformation contacted parts of the core that are distant in the X-ray structure. Because NMR experiments suggest that the catalytic core of the hammerhead was highly dynamic in solution (Bondensgaard et al. 2002), it seemed possible that the active conformation forms only transiently and the major conformation in solution is inactive and resembles the crystal structure.

The discovery by Khvorova et al. (2003) that the hammerhead contains a peripheral loop-loop interaction can potentially reconcile the structure-function dilemma. The presence of this stabilizing tertiary interaction could reduce the possible modes of motion available to the catalytic core, permitting the active conformation to form more often and resulting in the observed faster cleavage rate. As the role of the tertiary interaction is only to provide stability and not to participate in the cleavage mechanism, there is no need for it to be conserved. The suggestion by Wang et al. (1999) that a magnesium ion is used to stabilize the active configuration agrees nicely with the observation that the natural hammerheads appear to bind magnesium better than those without the tertiary interaction. If the tertiary inter- 
action stabilizes the catalytic core in the active conformation, the metal ion binding site would be preorganized and thereby bind metals better. An important goal will be to determine the X-ray and solution structures of one of the natural hammerheads. Although reasonable models for the loop-loop interaction were proposed by Khvorova et al. (2003), it will be interesting to know to what degree the structure of the catalytic core differs from the current structures. Any differences may account for the faster cleavage and tighter magnesium binding.

An outsider to the hammerhead field would be curious to know how such a very basic fact about the size of an important catalytic RNA motif was missed for so long. As one of the participants in the early days of hammerhead research, I can offer the following historical perspective, which should also serve as a lesson for those contemplating boundary experiments.

The hammerhead motif was first spotted in R. Symons' lab in 1986 (Hutchins et al. 1986) as a set of conserved residues that surrounded the self-cleavage site in several pathogenic RNA genomes. In order to show that the hammerhead was sufficient for cleavage, Forster and Symons (1987) performed the critical boundary experiments on lucerne transient streak viroid RNA. Because the $3^{\prime}$ and $5^{\prime}$ ends of this RNA extended from Stem 3 of the hammerhead, the loop1-loop2 interaction was preserved. They found that as they made shorter RNAs, the extent of cleavage that occurred in the in vitro transcription reaction increased dramatically, presumably because the number of inactive alternative conformations decreased. By the time they had truncated to a 55-mer, cleavage was virtually complete in the transcription reaction, and the small amount of uncleaved material that could be recovered appeared to cleave instantly upon reincubation. Because further deletion disrupted Stem 3 and reduced activity, the minimal hammerhead was considered to be defined.

At about the same time, inspired by the hammerhead consensus, I used our newly developed method to transcribe RNA from synthetic DNA to prepare two oligonucleotides that could be combined to assemble a hammerhead through the formation of Stems 1 and 2 (Uhlenbeck 1987). However, the critical loop1-loop2 interaction was missing. When the two oligonucleotides were mixed, the resulting cleavage was both more efficient and much faster than that reported for the longer viroid RNAs, suggesting that a minimal motif had been identified. This view was confirmed by Haseloff and Gerlach (1988) using bimolecular cleavage experiments on another hammerhead that was also missing the tertiary interaction. The critical experiment that no one did was to carefully compare the cleavage properties of the minimal unimolecular hammerhead which contained the tertiary interaction with one of the minimal bimolecular hammerheads which did not. Because both cleaved rapidly and efficiently, they were considered equiva- lent. In the succeeding years the experimentally more convenient bimolecular hammerheads gained favor, and the importance of the loop1-loop2 interaction was missed.

So what are the lessons for future RNA cutdown artists?

1. Do not rely entirely on consensus sequences. Nearly every natural hammerhead uses a different loop1-loop2 interaction, making the tertiary contact difficult to spot.

2. Make sure a quantitative assay is available. The rapid cleavage rate and tight magnesium binding of the natural hammerhead are easy to detect now that we know to look for it.

3. Use different kinds of assays. The transient transfection assay was the key to the discovery of the tertiary interaction.

4. Perform the cutdown in different ways. By breaking the RNA chain in loop1 or loop2, the tertiary interaction does not form, making it impossible to detect.

5. Finally, as always, assume that alternative RNA conformations will form and complicate your life.

\section{REFERENCES}

Bondensgaard, K., Mollova, E.T., and Pardi, A. 2002. The global conformation of the hammerhead ribozyme determined using residual dipolar couplings. Biochemistry 41: 11532-11542.

Engelhardt, M., Doherty, E.A., Knitt, D.S., Doudna, J.A., and Herschlag, D. 2000. The P5abc peripheral element facilitates preorganization of the tetrahymena group I ribozyme for catalysis. Biochemistry 39: 2639-2651.

Forster, A.C. and Symons, R.H. 1987. Self-cleavage of plus and minus RNAs of a virusoid and a structural model for the active sites. Cell 50: 9-16.

Haseloff, J. and Gerlach, W. 1988. Simple RNA enzymes with new and highly specific endoribonuclease activities. Nature 334: 585-591.

Hutchins, C.J., Rathjen, P.D., Forster, A.C., and Symons, R.H. 1986. Self-cleavage of plus and minus RNA transcripts of avocado sun blotch viroid. Nucleic Acids Res. 14: 3627-3640.

Khvorova, A., Lescoute, A., Westhof, E., and Jayasena, S.D. 2003. Sequence elements outside the hammerhead ribozyme catalytic core enable intracellular activity. Nat. Struct. Biol. 10: 708-712.

McKay, D.B. 1996. Structure and function of the hammerhead ribozyme: An unfinished story. RNA 2: 395-403.

Miller, W. and Silver, S. 1991. Alternative tertiary structure attenuates self-cleavage of the ribozyme in the satellite RNA of barley yellow dwarf virus. Nucleic Acids Res. 19: 5313-5320.

Pley, H.W., Flaherty, K.M., and McKay, D.B. 1994. Three-dimensional structure of a hammerhead ribozyme. Nature 372: 68-74.

Ryder, S.P. and Strobel, S.A. 2002. Comparative analysis of hairpin ribozyme structures and interference data. Nucleic Acids Res. 30: 1287-1291.

Scott, W., Finch, J., and Klug, A. 1995. The crystal structure of an all-RNA hammerhead ribozyme: A proposed mechanism for RNA catalytic cleavage. Cell 81: 991-1002.

Symons, R.H. 1992. Small catalytic RNAs. Annu. Rev. Biochem. 61: 641-671.

Uhlenbeck, O.C. 1987. A small catalytic oligoribonucleotide. Nature 328: 596-600.

Wang, S., Karbstein, K., Peracchi, A., Beigelman, L., and Herschlag, D. 1999. Identification of the hammerhead ribozyme metal ion binding site responsible for rescue of the deleterious effect of a cleavage site phosphorothioate. Biochemistry 38: 14363-14378. 

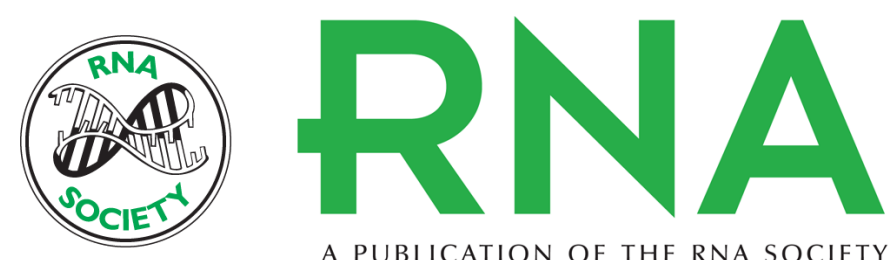

A PUBLICATION OF THE RNA SOCIETY

\section{Less isn't always more}

OLKE C. UHLENBECK

RNA 2003 9: 1415-1417

\section{License}

Email Alerting Receive free email alerts when new articles cite this article - sign up in the box at the Service top right corner of the article or click here.

To subscribe to $R N A$ go to:

http://rnajournal.cshlp.org/subscriptions 